\title{
Alpha-1-Acid Glycoprotein (AGP) as a Potential Biomarker for Breast Cancer
}

\author{
Kevin D. Smith, Jennifer Behan, \\ Gerardine Matthews-Smith and Anthony M. Magliocco
}

Additional information is available at the end of the chapter

http://dx.doi.org/10.5772/48177

\section{Introduction}

The majority of plasma proteins are modified by the addition of oligosaccharide chains (glycans) to their surface. The process, known as glycosylation, is responsible for introducing huge structural variation and is important in the determination of functional properties expressed by the overall glycoprotein. Glycan composition and structure can vary widely, potentially showing disease- specificity, unlike the underlying polypeptide sequence [1-3]. Therefore analysis of their structures could offer more accurate and condition-specific markers which is critical when early treatment and monitoring is crucial in tackling diseases. The monomeric units of glycans are the monosaccharides, linked together via glycosidic bonds between the $-\mathrm{OH}$ reducing group of $\mathrm{C} 1$ and any other $-\mathrm{OH}$ of adjacent residues (a condensation reaction). The bonds exist in either an $\alpha$ or $\beta$ anomeric configuration, depending on the orientation of the bond, providing further opportunity to generate structural variability.

Unlike the structure of the polypeptide backbone, glycosylation is not directly encoded by specific genes, but is reliant upon the concerted action of a series of highly specific enzymes (glycosyltransferases and glycosidases), which are resident in the ER and Golgi of cells, to initiate and processing glycan chain precursors. Any alterations in the expression of the genes encoding these enzymes due to, for example a pathophysiological condition, will affect the structure and composition of the glycans generated. During the synthesis and processing of glycans, they are transferred by the enzyme oligosaccharyltransferase from a lipid-linked oligosaccharide donor to polypeptide chains, either at asparagine (N-linked) or Serine/Threonine (O-linked) residues. N-linked glycans share a common pentasaccharide core (Man3GlcNAc2), however the overall structures can vary widely, primarily differing by the sequence and quantity of monosaccharides of which they are composed. Glycan synthesis then proceeds by the sequential addition/removal of individual monosaccharide units. 
Glycan expression is cell and tissue specific and dependent on the presence of (patho)physiological conditions [4]. Abnormal expression of even a single enzyme participating in this process may alter the subsequent steps and give rise to aberrant oligosaccharide structures. The cell types containing these biomolecules determine the enzymes expressed and therefore the glycans - variation reflects these source cells or tissue and the physiological and biochemical conditions present [3-4]. For example, abnormal or unusual glycan moieties are often detected on the surface of tumour cells, as well as among secreted glycoproteins. Many well known tumour-associated antigens are glycolipids or glycoproteins that contain aberrant glycan structures [5].

The post translational modification of proteins with oligosaccharide chains offers several advantages as a potential source of cancer biomarkers [5-6]. While protein biomarkers frequently occur in low abundance, distinctive cellular glycan structures, including those that are tumour-specific, are typically fairly abundant. Such structures are usually synthesised in multiple copies on a single glycoprotein molecule. In addition, the same tumour-specific glycan structures may be present on several different glycoproteins. Importantly, many currently used biomarker molecules, such as prostate specific antigen, CA125, and carcinoembryonic antigen, are monitored solely based on protein levels, not on their tumour-specific glycan moieties.

Glycoproteins with modified glycosylation resulting from the presence of cancer may be identifiable in body fluids that are typically amenable for clinical testing [6]. Alpha - 1 (or $\alpha 1$ ) - acid glycoprotein (AGP, or orosomucoid) is an important example of a naturally occurring N-linked plasma glycoprotein secreted by liver parenchymal cells. It was first isolated and characterised in the 1950s and although its specific biological role/function has yet to be clearly defined it is considered a natural anti-inflammatory and immunomodulatory agent [7-9], the concentration and/or 'normal' glycosylation of which may change under various physiological and pathophysiological conditions. It is a highly glycosylated $(45 \%)$ protein and therefore investigations into alterations in its glycan profile during different diseases has been, and is still, of great interest (Figure 1). Sialic acid, in the form of $\mathrm{N}$-acetylneuraminic acid (NeuAc), accounts for $12 \%$ of the total number of monosaccharides, and is responsible for giving AGP its low isoelectric point of 2.7.

Alterations in the protein levels of AGP have been well documented for numerous physiological and pathophysiological conditions including lung and breast cancer [10] and malignant mesothelioma [11]. It has been suggested that a marked increase in AGP concentration may limit adverse reactions such as inflammation by providing a form of negative feedback [12]. Increased concentrations are associated with expression of the ORM1 and ORM-1 genetic variants of AGP; however the proportions of each do not necessarily differ to that of a healthy individual [10]. In terms of plasma concentration, increased levels of AGP have been detected in the plasma of patients with breast cancer [13] and have also been shown to increase with disease progression [14] but to return to normal upon treatment with tamoxifen [15]. This indicates that the prognosis of breast cancer appears to be linked to the APR in general and AGP levels in particular. Not only is there a dearth of studies determining the levels of AGP in breast cancer but more importantly there has been 
no investigations of the glycosylation of AGP in breast cancer, the extent that it is altered and/or its significance as a diagnostic marker.

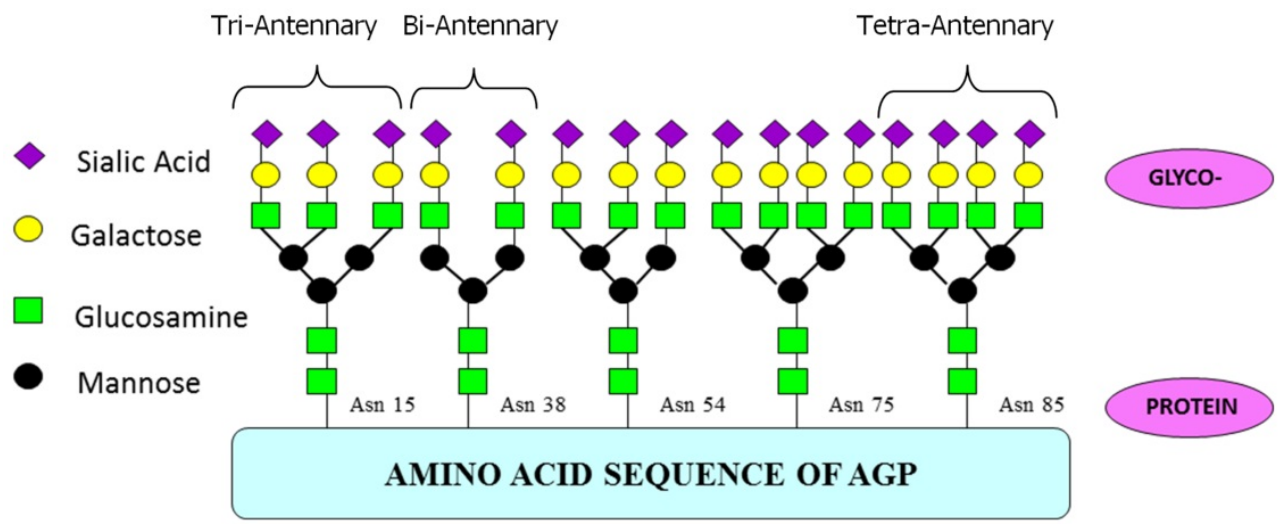

Figure 1. General schematic illustrating the structure of alpha-1-acid glycoprotein.

Although the concentration of AGP alone is not diagnostic for a particular pathological condition, the altered glycosylation of AGP (microheterogeneity) in different diseases, provides an alternative biomarker target. These alterations have the potential to be markers for particular diseases and also disease progression. The major form of heterogeneity, type I, is associated with a reduction in the number of branches on the oligosaccharide chains [4, 16-17]; the minor form (type II) concentrates on the composition of the oligosaccharide chains namely the extent of fucosylation and sialylation of the five oligosaccharide chains [18].

There is potential for the existence of $10^{5}$ different glycoforms of AGP due to the huge structural variability provided mainly by the presence of glycans. However in normal, nonpathological conditions there are only 12-20 expressed, each exhibiting various degrees of branching, fucosylation and sialylation [18]. The number of feasible glycoforms is reduced because the asparagines (Asn) residues are selective for the type of glycans they express in terms of the degree of branching. The first and second Asn sites (Asn 15 and 38) prefer to harbour bi-antennary glycans whilst Asn 15 will not bind a tetra-antennary chain and Asn 38 never binds fucosylated glycans. Conversely, Asn 75 and Asn 85 prefer more branched glycans; in fact site Asn 75 never carries bi-antennary chains and Asn 85 usually expresses the greatest degree of $\alpha 1,3$-fucosylation [16]. This increased branching could explain why only these two sites potentially carry a tetra-antennary chain with more than one fucose residue [8]. It has been found that the majority of AGP glycans have a tri/tetra-antennary structure $(85-90 \%)$ and the remaining $10-15 \%$ is bi-antennary glycans [19]. Sialic acid can be bound $\alpha 2-3$ or $\alpha 2-6$ on terminal galactose residues; fucose can be bound $\alpha 1-3$ to an external $\mathrm{N}$-acetlyglucosamine and also by $\alpha 1-6$ and $\alpha 1-2$ to core $\mathrm{N}$-acetlyglucosamine and galacose residues respectively [8]. The extent of fucosylation in a healthy population varies with $30 \%$ [8] to $40 \%$ [20] containing no fucose. 
Altered glycosylation of AGP has been widely studied in a number of pathophysiological conditions. Decreased branching of the five glycans of AGP has been demonstrated in acute inflammation while an increase is associated with chronic inflammation [21-23]. Chronic inflammatory states such as those found in patients with RA are characterised by an increase in branching [24, 25], increased fucosylation of the chains [26-31] and increased sialic acid content [32-33]. The expression of the antigen sialyl lewis X $\left(\mathrm{SLe}^{\mathrm{X}}\right)$ has been found to increase on AGP oligosaccharides [20,25-26] at any one of the 5 sites of glycosylation [27]. SLe $^{\mathrm{x}}$ is normally expressed on white blood cells and aids through binding to the endothelial ligand, E-Selectin, the extravasation of leukocytes into tissues to mount their inflammatory response. De Graaf and colleagues [20] proposed that the increased expression of this antigen on AGP represents a negative feedback response during inflammatory conditions. Further work by Jørgensen et al. [26] using a microtitre cell-protein binding assay discovered that SLe ${ }^{\mathrm{x}}$-containing AGP expressed in patients with RA was able to inhibit binding of SLe ${ }^{\mathrm{x}}$ presenting cells to E-Selectin. Thus, during inflammatory states such as those in RA, the abnormally glycosylated form of AGP has the ability to competitively bind to E-Selectin and inhibit leukocyte extravasation exerting an overall anti-inflammatory effect. Highly branched structures have also been noted in individuals with liver disease, along with increased fucosylation of these chains [34-36]. Increased concentrations of AGP were found in the cerebrospinal fluid (CSF) of patients with multiple sclerosis (MS) compared to disease free individuals [37] and in the plasma/serum of patients with burns injuries [38]. It is known that various factors act to influence AGP glycosylation including the congentital disorders [28], pregnancy [29, 33], drug or glucocorticoid use [39], and oral contraceptive use [40].

Investigations into the heterogeneity of AGP in cancer have found an increase in biantennary glycan content with increased fucosylation and sialylation. An early study by [41] reported that the major microheterogeneity of AGP from malignant, benign and normal groups was significantly different from each other. When compared with normal controls, sera from patients with inflammatory lung disease (benign group) exhibited an increase in tri-and tetra-antennary glycans and in sera from lung cancer patients (malignant group) there was an increase in bi-antennary expression. This work was contradicted the following year in an investigation [42] which repeated the study including an evaluation of the microheterogeneity of AGP in colorectal cancer and reported that there was no significant difference in the microheterogeneity of AGP from malignant and non-malignant inflammatory disease. Evidence to support the cancer-induced expression of bi-antennary glycans on AGP detailed by Hansen and colleagues was provided by [43] who reported a similar finding on AGP from the ascitic fluid of liver cancer patients.

Reports of changes in the minor microheterogeneity of AGP from studies of the oligosaccharide content of the chains in cancer have been detailed. Early studies [44-45] observed an increase in the sialic acid content of cancer AGP glycans compared to normal controls. A more recent study by Hashimoto et al. [46] on AGP branching and fucosylation in cancer concluded that changes in microheterogeneity could be used as a marker of carcinoma progression and prognosis. Patients with advanced stages of the disease who 
displayed highly branched glycans with a high fucose content for a significant duration post-surgery were associated with poor prognosis whereas, patients who did not display this increase in branching and fucosylation were likely to have a good, more reassuring prognosis. When highly branched and fucosylated glycans were present a prolonged period after surgery, a poor prognosis was associated. Similar analyses can be associated with surgery and other traumas in general [47].

\section{Problem statement}

The majority of studies [reviewed in 7-9] have concluded that, although increased levels of AGP have been detected in the plasma of patients with breast cancer, this concentration is largely unrelated to disease progression. The prognostic value of AGP glycosylation in breast cancer is still largely unproven. Although the limited research to date has discovered structural differences in the glycosylation in AGP isolated from different cancers and early and advanced stages of the same cancer, very few studies have looked at breast cancer samples. We hypothesise that alpha-1-acid glycoprotein (AGP), which is a common constituent in all blood, could be a diagnostic marker for early breast cancer.

\section{Application area}

There is a critical need for information regarding a reliable biomarker that could be used to predict the onset, severity, progression and prognosis of breast cancer. The identification of alterations in AGP glycosylation specific to individual stages of breast cancer could, through being altered to the "normal" healthy AGP glycosylation pattern, result in the development of a diagnostic test based on AGP glycosylation for the onset, progression and/or prognosis of breast cancer.

\section{Aims of study}

To determine whether specific alterations in the glycosylation pattern of alpha-1-acid glycoprotein (AGP) could be diagnostic for the for the onset, progression and/or prognosis of breast cancer.

\section{Study design and methodology (Figure 2)}

\subsection{Patient samples}

Breast cancer can occur as a non-malignant or malignant tumour. Non-malignant cancers can be further subdivided into ductal carcinoma in situ (DCIS) and lobular carcinoma in situ (LCIS). In situ refers to the state and location of the breast epithelial cells in that they have gone through malignant transformation and are now proliferating but remain at the site of origin and do not penetrate the basement membrane into surrounding tissues. DCIS and LCIS are not at risk of metastatic spread as there are no blood vessels or lymphatics in the epithelial layer of the breast [48]. The two most common types of invasive or malignant 
breast cancer are Invasive ductal carcinoma (IDC) and Invasive lobular carcinoma (ILC). Approximately $75 \%$ of breast cancers are IDC with ILC accounting for a further $10 \%$. The remaining $15 \%$ of diagnosed invasive breast cancers are made up of rare types such as mucinous, medullary, tubular, and papillary. Blood samples were taken from non-fasting patients with consent by Calgary Laboratory Services. The blood samples were drawn into $5 \mathrm{~mL}$ Red Top tubes and processed within 30 minutes. During processing, the blood samples were spun for 30 minutes to separate them into components. The samples were stored at -80 degrees Celsius. The patient details are given in Table 1.

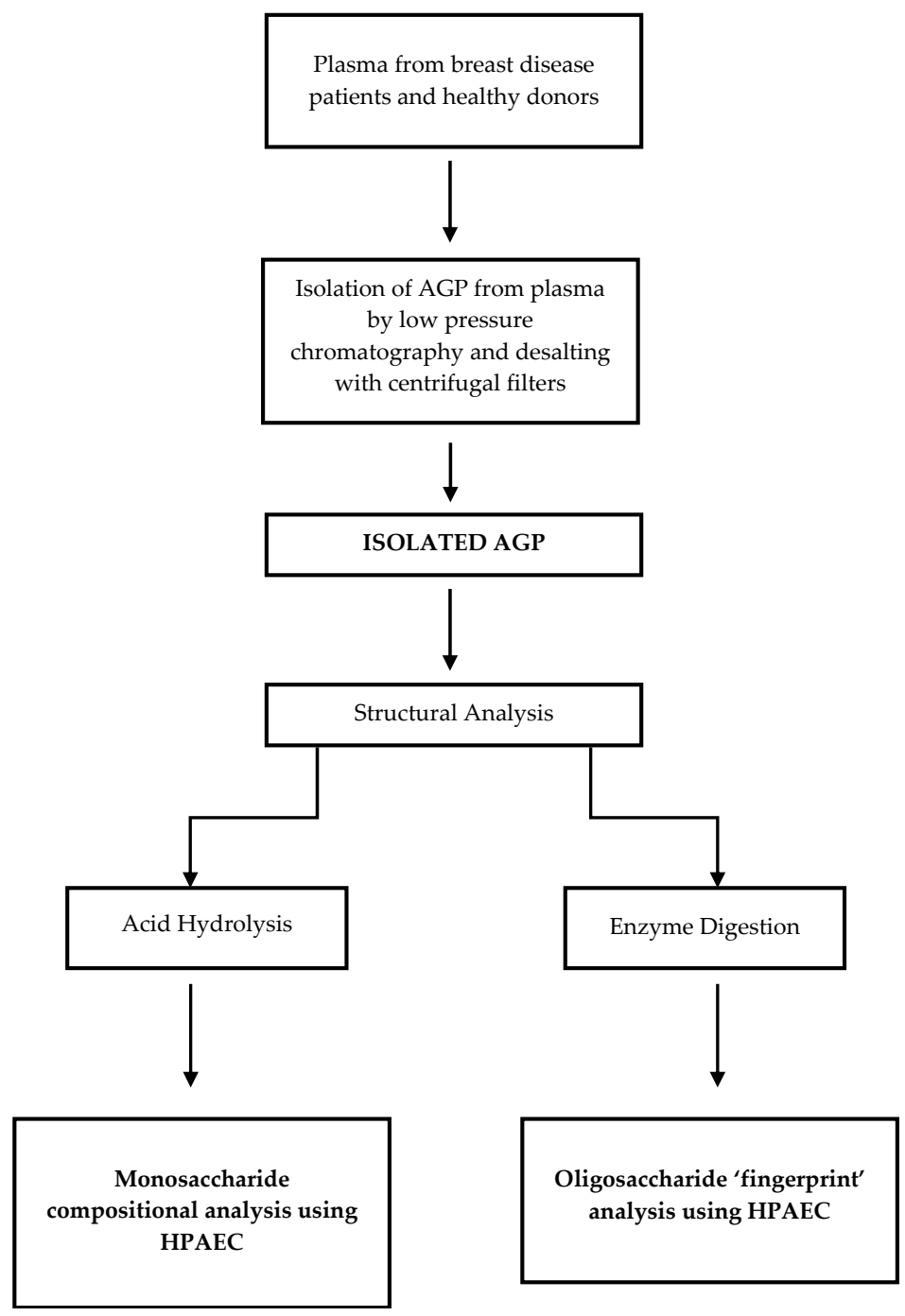

Figure 2. The design of the study to determine the glycosylation patterns of AGP in breast cancer 


\begin{tabular}{|c|c|c|c|c|c|c|}
\hline No & Age & Description & Her2 & Malignant. & Grade & ER \\
\hline 1 & 62 & Benign proliferative breast disease & N/A & No &. &. \\
\hline 2 & 78 & DCIS (+ invasive ductal carcinoma) & neg. & Yes & 3.00 &. \\
\hline 3 & 48 & Benign proliferative breast disease & N/A & No &. &. \\
\hline 4 & 77 & Invasive duct carcinoma & neg. & Yes & 1.00 & 1.00 \\
\hline 5 & 51 & Invasive duct carcinoma & neg. & Yes & 2.00 & 1.00 \\
\hline 6 & 35 & Invasive duct carcinoma & neg. & Yes & 1.00 & 1.00 \\
\hline 7 & 39 & Benign proliferative breast disease & N/A & No &. &. \\
\hline 8 & 35 & Malignant phyllodes tumor. & neg. & Yes &. &. \\
\hline 9 & 53 & DCIS (+ invasive ductal carcinoma) & neg. & Yes &. &. \\
\hline 10 & 49 & Invasive duct carcinoma & pos. & Yes & 2.00 & 1.00 \\
\hline 11 & 76 & Invasive duct carcinoma & neg. & Yes & 2.00 & 1.00 \\
\hline 12 & 65 & Invasive duct carcinoma & neg. & Yes & 3.00 & 1.00 \\
\hline 13 & 32 & Invasive ductal carcinoma & pos. & Yes &. &. \\
\hline 14 & 43 & Invasive duct carcinoma & neg. & Yes & 3.00 & 1.00 \\
\hline 15 & 40 & Biphasic fibroepithelial lesion & N/A & No &. &. \\
\hline 16 & 48 & Benign proliferative breast disease & N/A & No &. &. \\
\hline 17 & 50 & Invasive duct carcinoma & pos. & Yes & 3.00 & 1.00 \\
\hline 18 & 69 & Invasive duct carcinoma & neg. & Yes & 2.00 &. \\
\hline 19 & 45 & Invasive duct carcinoma & pos. & Yes & 3.00 & .00 \\
\hline
\end{tabular}

Table 1. Demographic and clinical details of patients used in study

\subsection{Materials}

\subsubsection{AGP Isolation}

Polyethylene glycol (PEG 3350), the low pressure chromatographic material (Cibacron Blue 3GA, Q-sepharose and Red Sepharose CL-6B), potassium thiocyanate, sodium acetate, sodium chloride and Trizma base were purchased from Sigma (Poole, UK). Bio-Rad, Hemel Hampstead, UK, provided the poly-prep disposable 10ml columns. HPLC-grade water and the Centricon YM10 centrifugal filter device were purchased from Rathburn Chemicals (Walkerburn, UK) and Millipore (Bedford, USA) respectively.

\subsubsection{High $p H$ anion exchange chromatography monosaccharide analysis}

HPAEC was carried out on a DX600 system supplied by Dionex (Camberley, UK), consisting of a GP50 gradient pump and an ED40 electrochemical detector, controlled with PeakNet software via a Dell OptiPlex GX110 personal computer. Separation of monosaccharides was achieved using a CarboPac PA-100 (250x4mm) and guard column (50x4mm). HPLC grade water was purchased from Rathburn Chemicals, Walkerburn, UK, and sodium hydroxide $50 \% \mathrm{w} / \mathrm{v}$ was obtained from BDH, Poole, UK. The monosaccharide standards and Dowex-50W were purchased from Sigma, Poole, UK. HPLC grade trifluoroacetic acid was purchased from Perbio Science, UK Ltd, Talenhall, UK. 


\subsubsection{Oligosaccharide analysis}

PNGase F and buffers (NP40 and NE buffer G7) were supplied by New England BioLabs (New England, USA). HPAEC was carried out on a DX500 system supplied by Dionex (Camberley, UK), consisting of a GP40 gradient pump and an ED40 electrochemical detector, controlled with PeakNet software via a Vtech personal computer. Separation of monosaccharides was achieved using a CarboPac PA-100 $(250 \times 4 \mathrm{~mm})$ and guard column (50x4mm). HPLC grade water was purchased from Rathburn Chemicals, Walkerburn, UK, and sodium hydroxide $50 \% \mathrm{w} / \mathrm{v}$ was obtained from BDH, Poole, UK. Sodium acetate was purchased from Sigma (Poole, UK).

\subsection{Methods}

\subsubsection{Polyethylene Glycol (PEG) precipitation}

First, PEG 3350 was added to each plasma sample to a final concentration of $40 \% \mathrm{w} / \mathrm{v}$ in a microcentrifuge tube. This was then mixed vigorously for ten minutes before being stored overnight at $4^{\circ} \mathrm{C}$. Subsequently the mixture was centrifuged at $14,000 \mathrm{rpm}$ for 30 minutes. The supernatant was removed to a clean eppendorf and frozen until required, with the pellet being discarded.

\subsection{Low pressure chromatography to isolate AGP}

A disposable BioRad low-pressure chromatography column $(10 \mathrm{ml})$ was packed to a bed volume of approximately $6 \mathrm{ml}$ with the appropriate chromatographic resin. Several volumes of the appropriate Elution buffer (Table 2) was washed through the column using a Pharmacia LKB peristaltic pump at a flow rate of $0.5 \mathrm{ml} / \mathrm{min}$, with the change in UV absorbance at $280 \mathrm{~nm}$ being detected using a Pharmacia LKB optical unit. Peak fractions were collected in $15 \mathrm{ml}$ disposable centrifuge tubes and dried under vacuum to approximately $2 \mathrm{ml}$ between stages, using a centrifugal evaporator. $2-3 \mathrm{ml}$ of the PEGprecipitated serum sample is first applied to a Cibacron blue column, to which the bilirubin site of albumin binds. The remaining soluble proteins are eluted with $0.05 \mathrm{M}$ Tris/0.1M potassium chloride/0.02\% Azide ( $\mathrm{pH} 7.0$ ) elution buffer while the albumin is removed from the column with $0.5 \mathrm{M}$ potassium thiocyanate. One peak is collected upon elution. After drying the peak is next applied to the ion-exchange column Q-sepharose. This divides the eluate from the previous column into three fractions; $\alpha_{1}$-antitrypsin, AGP and transferrin. A column gradient of $0.075-0.5 \mathrm{M}$ sodium chloride $(\mathrm{pH} 6.5)$ is utilised, and the second peak, which contains AGP, is collected. The column is regenerated between samples by washing the column with $0.075 \mathrm{M} \mathrm{NaCl}$ buffer until a flat baseline is observed. The AGP is then, after again drying to $2-3 \mathrm{ml}$, applied to a column containing the Red sepharose resin in order to remove any trace proteins that may remain. The AGP in the sample is eluted with a $0.03 \mathrm{M}$ sodium acetate elution buffer and collected, while the bound proteins are removed with $1 \mathrm{M}$ sodium chloride. The AGP peak is then dried to $2 \mathrm{ml}$ in preparation for desalting. 


\begin{tabular}{|c|c|c|}
\hline & Buffer & \\
\hline Chromatography resin & Elution & Desorption \\
\hline Cibacron Blue & $\begin{array}{l}\text { 50mM Trizma/0,1mM } \\
\mathrm{KCl} / 0.02 \% \text { Azide at } \mathrm{pH} 7.0\end{array}$ & $\begin{array}{l}\text { 500mM KSCN/50mM } \\
\text { Trizma/0.1mM KCl/0.02\% } \\
\text { Azide pH } 7.0\end{array}$ \\
\hline Q-sepharose fast flow & $\begin{array}{l}\text { 20mM Trizma/75mM NaCl } \\
\text { pH6.5 }\end{array}$ & $\begin{array}{l}\text { Equilibrium buffer: } 20 \mathrm{mM} \\
\text { Trizma/75mM NaCl pH } 6.5 \\
\end{array}$ \\
\hline Red sepharose CL-6B & $30 \mathrm{mM} \mathrm{NaOAc}$ & $1 \mathrm{M} \mathrm{NaCl} / 30 \mathrm{mM} \mathrm{NaOAc}$ \\
\hline
\end{tabular}

Table 2. Buffers for each stage of low-pressure chromatography

\subsection{Desalting}

In The AGP peak from red sepharose was subsequently desalted using centrifugal ultrafiltration. $2 \mathrm{~mL}$ of the sample was added to a Centricon centrifugal filter device. This removes any salt in the solution and also acts to concentrate the AGP. The centricons were spun at $4000 \mathrm{rpm}$ until the filtrate had passed entirely through the membrane into the filtrate vial. To ensure complete removal of salt from the sample $1 \mathrm{ml}$ of HPLC water was added to the sample reservoir and spun as before. The centricon was then inverted and spun for 10 minutes at $1000 \mathrm{rpm}$, transferring the concentrate into the collection vial. The desalted glycoprotein is then transferred to an eppendorf and dried down to completion using a centrifugal evaporator.

\subsection{High pH Anion Exchange Chromatography (HPAEC)}

\subsubsection{Preparation of monosaccharides: Acid Hydrolysis of AGP}

Approximately $50 \mu \mathrm{g}$ of isolated AGP was hydrolysed using $100 \mu \mathrm{l} 2 \mathrm{M}$ trifluoroacetic acid (TFA) and $50 \mu \mathrm{l} 4 \mathrm{M}$ hydrochloric acid in a reacti-vial then sealed with a Teflon disc. The reacti-vial was then placed on a heating block pre-heated to $100^{\circ} \mathrm{C}$ for four hours in order to reach optimum hydrolysis. The monosaccharides were separated from the peptide fragments by eluting the hydrolysate down a Dowex-50W cation exchange column. A Pasteur pipette was plugged with glass wool and loaded with the resin to a bed volume of $1 \mathrm{ml}$. Each AGP sample was added to a separate column and washed through with HPLC water. The neutral monosaccharides were washed through and collected, then dried to completion in preparation for analysis.

\subsubsection{Monosaccharide analysis}

Monosaccharide analysis was carried out using a DX600 ${ }^{\mathrm{TM}}$ system. Separation of the monosaccharides, as their oxyanions, was performed at $\mathrm{pH} 13$ on a CarboPac PA-100 column. The monosaccharides were resolved using an isocratic elution with $30 \mathrm{mM}$ $\mathrm{NaOH}$, at a flow rate of $0.5 \mathrm{ml} / \mathrm{min}$ for 35 minutes. The column was regenerated after each run with $0.5 \mathrm{M} \mathrm{NaOH}$ for 5 minutes, prior to equilibration with $20 \mathrm{mM} \mathrm{NaOH}$ for a further 
10 minutes. Detection of monosaccharides was by pulsed amperometric detection (PAD) at the following pulse potentials: 0 sec: $E=0.05 \mathrm{~V} ; 0.29$ sec: $E=0.05 \mathrm{~V} ; 0.49$ sec: $\mathrm{E}=0.05 \mathrm{~V}$; 0.50sec: $\mathrm{E}=0.05 \mathrm{~V}$; 0.51sec: $\mathrm{E}=0.6 \mathrm{~V} ; 0.6 \mathrm{sec}$ : $\mathrm{E}=0.6 \mathrm{~V} ; 0.61$ sec: $\mathrm{E}=-0.6 \mathrm{~V} ; 0.65 \mathrm{sec}: \mathrm{E}=-0.6 \mathrm{~V} ; 0.66 \mathrm{sec}$ : $\mathrm{E}=0.05 \mathrm{~V}$ (Figure 3).

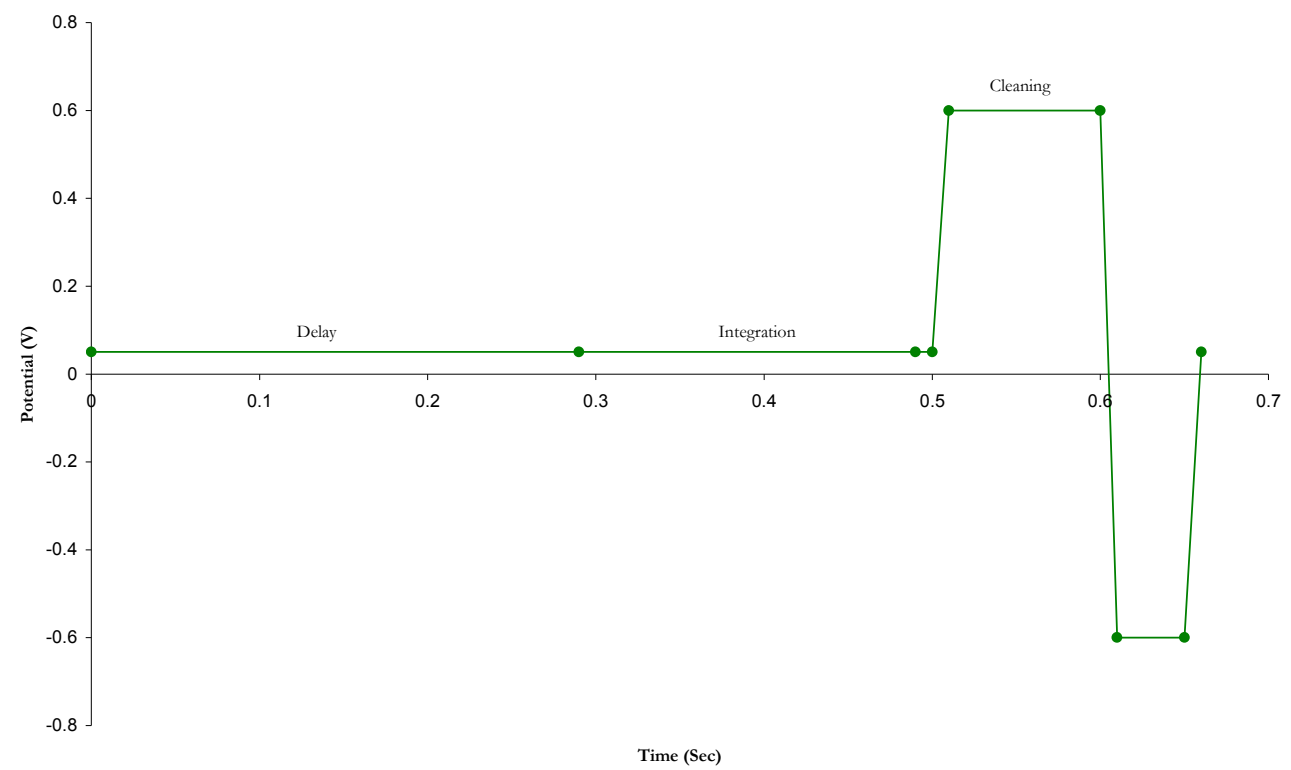

Figure 3. The triple pulsed waveform used in pulsed amperometric detection

An internal standard (IS) of 2-deoxy-D-galactose was utilised to determine the elution position of the monosaccharides by dividing the elution time of the IS by the time of the unknown peak. The calculated ratio can then be compared to known standards.

\subsubsection{HPAEC-PAD oligosaccharide analysis}

The required amount (50-100 $\mu$ g) of AGP was reconstituted in $100 \mu \mathrm{L}$ of HPLC grade water. The AGP solution was subsequently denatured through boiling for one hour. To the denatured AGP $10 \mu \mathrm{L}$ of NP-40, $10 \mu \mathrm{L}$ of NE Buffer G7 and 100U of PNGase F were added. PNGaseF cleaves the oligosaccharide chains from the AGP peptide moiety. The enzymatic reaction was allowed to proceed overnight while incubated at $37 \mathrm{C}$. Following incubation the solution was subjected to cold ethanol precipitation. $1 \mathrm{ml}$ of cold ethanol was added to each sample and then centrifuged at 10000rpm for 10 minutes which pelleted the peptide fractions and left the soluble oligosaccharides in the supernatant. Thus the supernatant was removed and dried to completion. 
Analysis of the enzymatically released oligosaccharides was performed using a DX500 system. Similar to monosaccharide analysis, oligosaccharides are eluted as their oxyanions, formed in the highly alkaline environment. Resolution is achieved using a CarboPac PA-100 column packed with a micro-pellicular resin. The column was equilibrated for 10 minutes with $10 \% 1 \mathrm{M}$ sodium hydroxide, 5\% $1 \mathrm{M}$ sodium acetate and $85 \%$ HPLC grade water. Following equilibration a linear gradient was developed over 40 minutes to a final eluant composition of $10 \%$ sodium hydroxide, $20 \%$ sodium acetate and $70 \%$ water. These conditions were maintained for 5 minutes before the column was regenerated for ten minutes with $50 \%$ sodium hydroxide and 50\% water. The pulsed amperometry was performed as described for the monosaccharide analysis.

\section{Results}

This investigation studied AGP from 19 breast cancer sufferers with either malignant or non malignant forms of breast cancer in term of the monosaccharide composition of the oligosaccharide chains and the oligosaccharide fingerprint of the intact chains themselves. Under alkaline conditions, the hydroxyl groups of a monosaccharide, either individually or as part of a sequence, are ionised to varying degrees such that they exist as negatively charged oxyanions. The varying location of the $\mathrm{OH}$ groups results in slight differences in the pKa value (ranging from 12-14) of individual monosaccharides under these conditions. These differences in ionisation, which are unique both with respect to individual monosaccharides and also cumulatively in terms of a sequence of monosaccharides, can be exploited in chromatographical terms using the anion exchange stationary phases and the electrochemical detection (based on pulsed potentials) components of high $\mathrm{pH}$ anion exchange chromatography (HPAEC). For monosaccharide analysis, an alkaline environment of $0.03 \mathrm{M}$ sodium hydroxide ionises monosaccharides in the hydrolysate and results in the formation of oxyanions unique to individual monosaccharides. The interaction of the charged oxyanions with the strong anionexchange CarboPac ${ }^{\mathrm{TM}}$ column and the slight differences in relative $\mathrm{pKa}$ values aids the chromatographic separation of individual monosaccharides. The monosaccharide compositions were determined, after acid hydrolysis, using high $\mathrm{pH}$ anion exchange chromatography (HPAEC). The results obtained are summarised in Table 3 and a typical HPAEC profile is shown in Figure 4.

The monosaccharide composition of the AGP oligosaccharide chains was found to differ between normal, non-malignant and malignant groups. Of all the samples containing fucose, the malignant breast cancer group displayed the highest average level of this monosaccharide compared to the normal and non malignant breast disease group. Only the malignant breast cancer population had $\mathrm{N}$-acetylgalactosamine present; this monosaccharide is not usually found in AGP and was completely absent from the normal samples analysed in the study. The graphs in Figures 5-7 illustrate the statistical significance of the fucose and Nacetylgalactosamine composition of AGP with respect to malignancy. 


\begin{tabular}{|c|c|c|c|c|}
\hline Patient & Description & Galactose $^{*}$ & Fucose $^{1}$ & GalNAc $^{1}$ \\
\hline 1 & Benign proliferative breast disease & 14.12 & 1.22 & .00 \\
\hline 2 & DCIS (+ invasive ductal carcinoma) & 15.58 & 1.78 & 1.25 \\
\hline 3 & Benign proliferative breast disease & 14.57 & .98 & .00 \\
\hline 4 & Invasive duct carcinoma & 16.22 & 3.12 & 2.87 \\
\hline 5 & Invasive duct carcinoma & 17.33 & 4.12 & 3.55 \\
\hline 6 & Invasive duct carcinoma & 16.47 & 2.95 & 1.14 \\
\hline 7 & Benign proliferative breast disease & 14.29 & 1.04 & .00 \\
\hline 8 & Malignant phyllodes tumor. & 18.29 & 4.56 & 3.22 \\
\hline 9 & DCIS (+ invasive ductal carcinoma) & 15.98 & 2.55 & .98 \\
\hline 10 & Invasive duct carcinoma & 17.78 & 4.01 & 3.68 \\
\hline 11 & Invasive duct carcinoma & 17.56 & 3.93 & 3.44 \\
\hline 12 & Invasive duct carcinoma & 19.91 & 6.02 & 4.45 \\
\hline 13 & Invasive ductal carcinoma & 18.84 & 5.10 & 4.06 \\
\hline 14 & Invasive duct carcinoma & 19.76 & 5.77 & 4.89 \\
\hline 15 & Biphasic fibroepithelial lesion & 11.75 & .76 & .00 \\
\hline 16 & Benign proliferative breast disease & 13.95 & 1.31 & .00 \\
\hline 17 & Invasive duct carcinoma & 19.82 & 5.52 & 4.36 \\
\hline 18 & Invasive duct carcinoma & 17.43 & 4.19 & 3.49 \\
\hline 19 & Invasive duct carcinoma & 19.64 & 5.59 & 4.80 \\
\hline
\end{tabular}

* units in $\mathrm{mol} / \mathrm{mol}$ AGP

Table 3. The monosaccharide compositions of oligosaccharide chains from AGP, isolated from healthy volunteers, patients with non malignant breast cancer and patients with malignant breast cancer, determined using high $\mathrm{pH}$ anion exchange chromatography

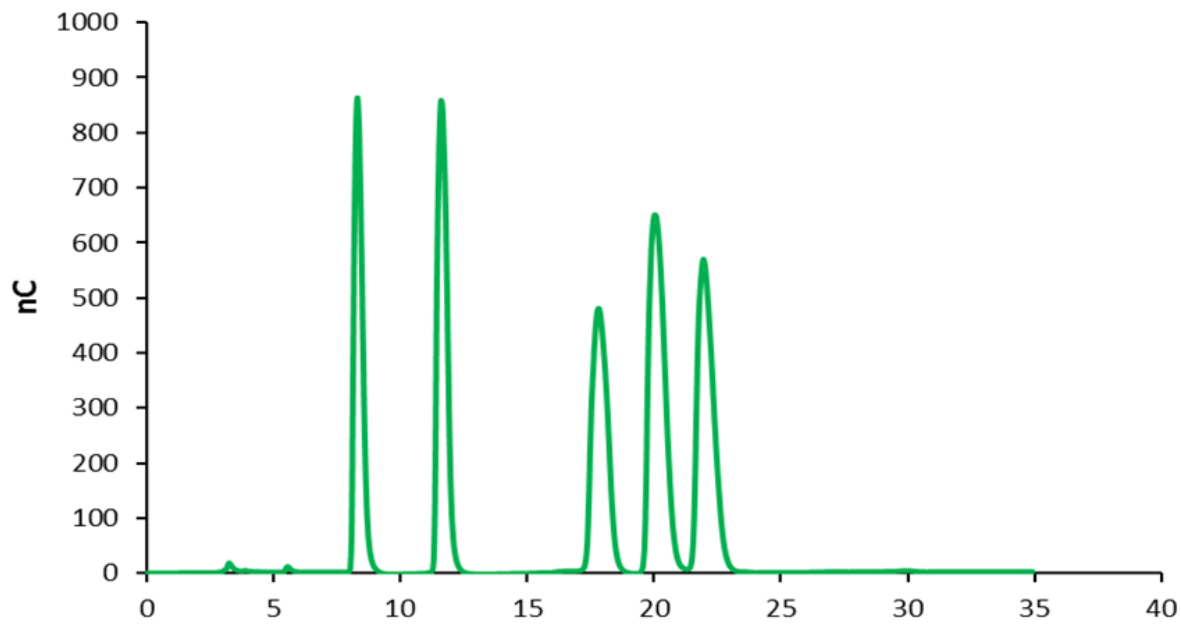

Time (mins)

Figure 4. The separation of a mixture of monosaccharides by high $\mathrm{pH}$ anion exchange chromatography 


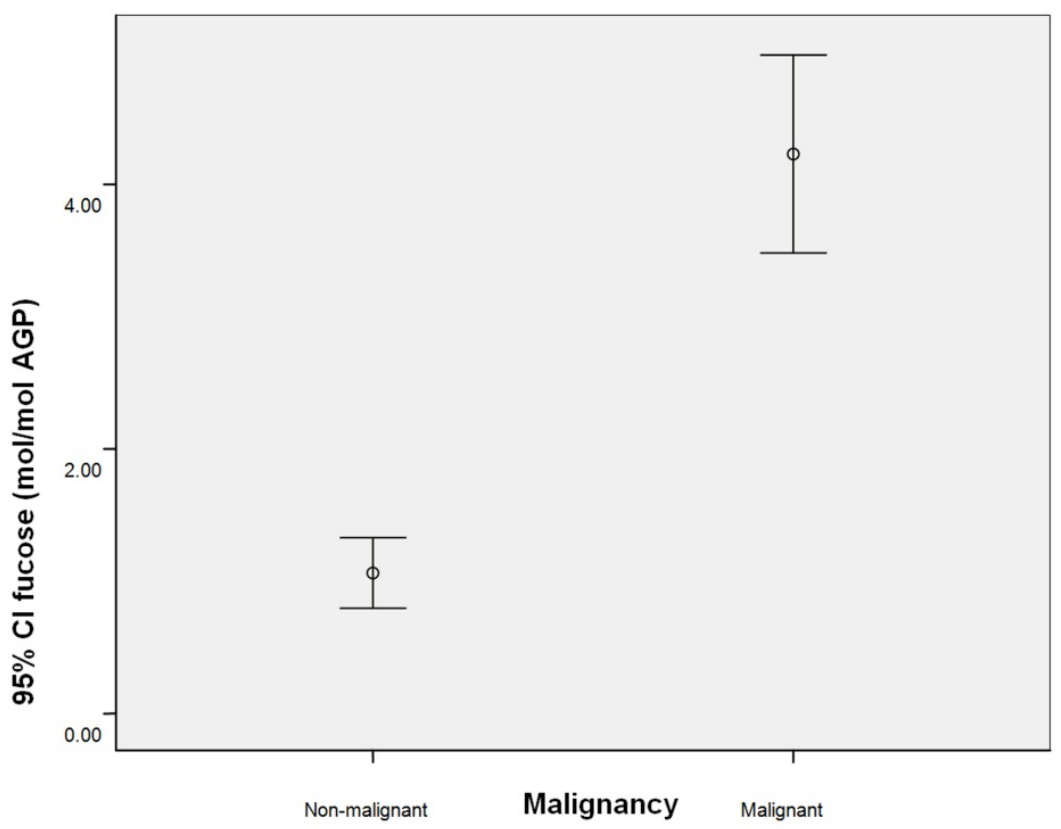

Figure 5. Fucose Composition of AGP with Respect to Malignancy

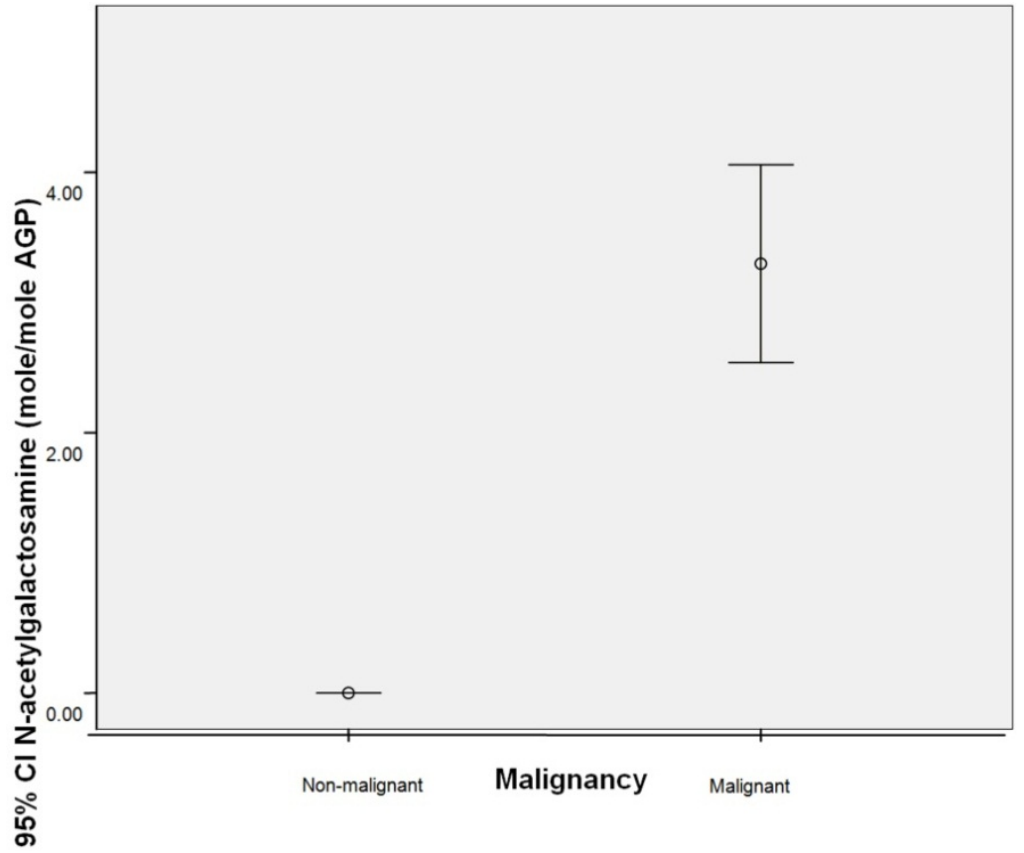

Figure 6. N-acetylgalactosamine Composition of AGP with Respect to Malignancy 


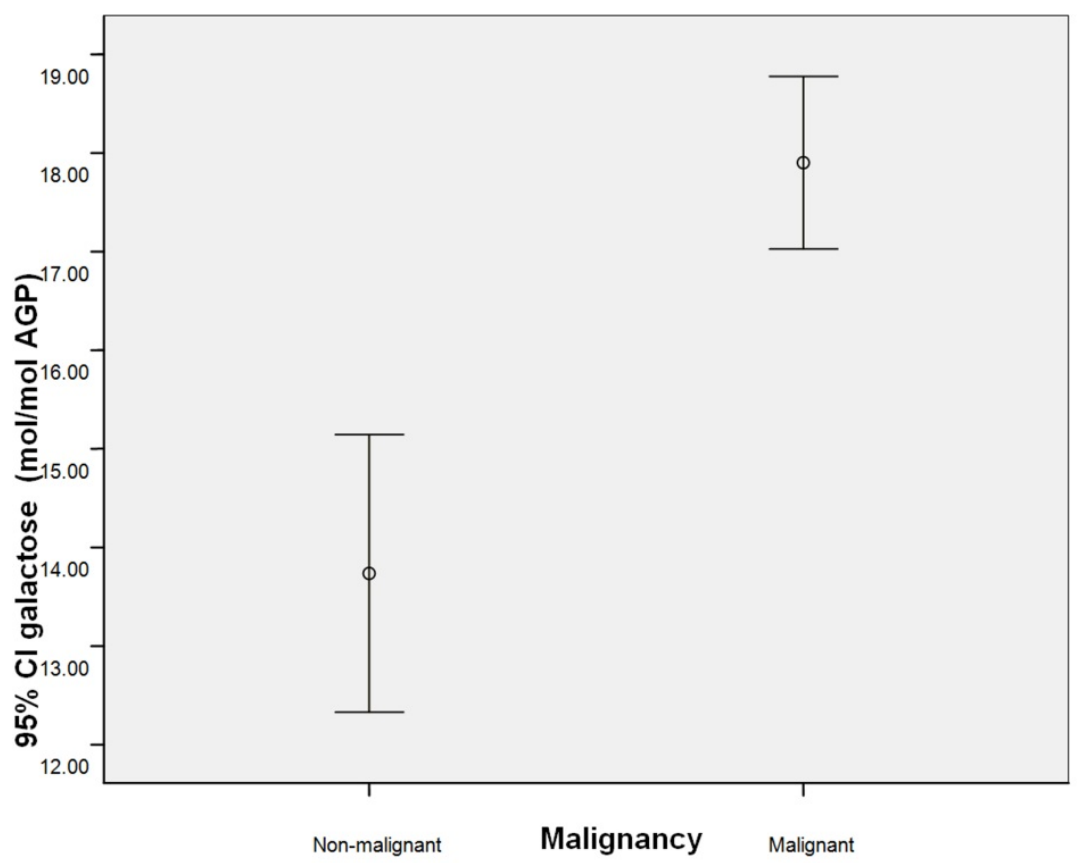

Figure 7. Galactose Composition of AGP with Respect to Malignancy

The monosaccharides fucose and $\mathrm{N}$-acetylgalactosamine are not normally found on AGP oligosaccharide chains; however, both were found to be present in plasma from breast cancer patients. Most importantly, the presence of $\mathrm{N}$-acetylgalactosamine appears to correlate with the presence of malignancy (Figure 6). While fucose appears to be present in small levels in patients with benign proliferative breast disease and in increased levels in malignant breast disease (Figure 5), $\mathrm{N}$-acetylgalactosamine appears to be present only in ductal carcinoma in-situ (DCIS) and invasive carcinoma. Each AGP molecule has five oligosaccharide chains, and each chain can have two, three, or four branches. Given that each branch contains a galactose molecule (refer to Figure 1), a higher degree of branching can be identified by a higher level of galactose. Therefore, increased galactose levels appear to be indicative of increased Malignancy (Figure 7). The AGP oligosaccharide profiles differed between the normal, non-malignant and malignant breast cancer groups.

The flexibility of HPAEC is illustrated by the fact that it also allows the efficient separation of oligosaccharide chains on the basis of their size, formal charge, monosaccharide composition and intra-chain linkages [49]. A major advantage over other techniques is that the HPAEC of oligosaccharides requires no prior derivatisation and can detect to picomole sensitivity. The separation of oligosaccharide structures using HPAEC is based primarily on the negative charge present on the oligosaccharides due to the presence of terminal sialic acid residues. The greater the number of sialic acid residues, the greater the negative charge. This leads to a stronger interaction between the oligosaccharide and the resin in the HPAEC 
analytical column. The application of a sodium acetate gradient causes the displacement of oligosaccharides, thus residues with a greater charge require a higher acetate concentration to cause displacement. Therefore, for example, a trisialylated oligosaccharide structure is retained longer than a bisialylated structure. Thus there is a positive correlation between the degree of negative charge and the elution time. Within charge bands, structures can be further separated on the basis of size i.e. a biantennary, bisialylated structure will be retained less than a triantennary, bisialylated oligosaccharide.

On a more subtle level the separation of oligosaccharides within each charge band is determined by the sequence of monosaccharides which form the chain and also the linkages between them. The involvement of hydroxyl groups in glycosidic bonds between monosaccharides can alter the charge of the sequence. Therefore oligosaccharides with the same monosaccharides but differing in one linkage can be separated using this technique. The analysis of oligosaccharides is qualitative, unlike monosaccharide analysis which is both qualitative and quantitative, allowing the pattern of oligosaccharides to be determined [4950]. The separation of a standard library of the oligosaccharide chains of AGP is shown in Figure 8. In the profile, each peak corresponds to a single oligosaccharide chain. The sialylated portion of oligosaccharide chains, such as those found in AGP, are separated into distinct charge bands based on the number of terminal sialic acids.

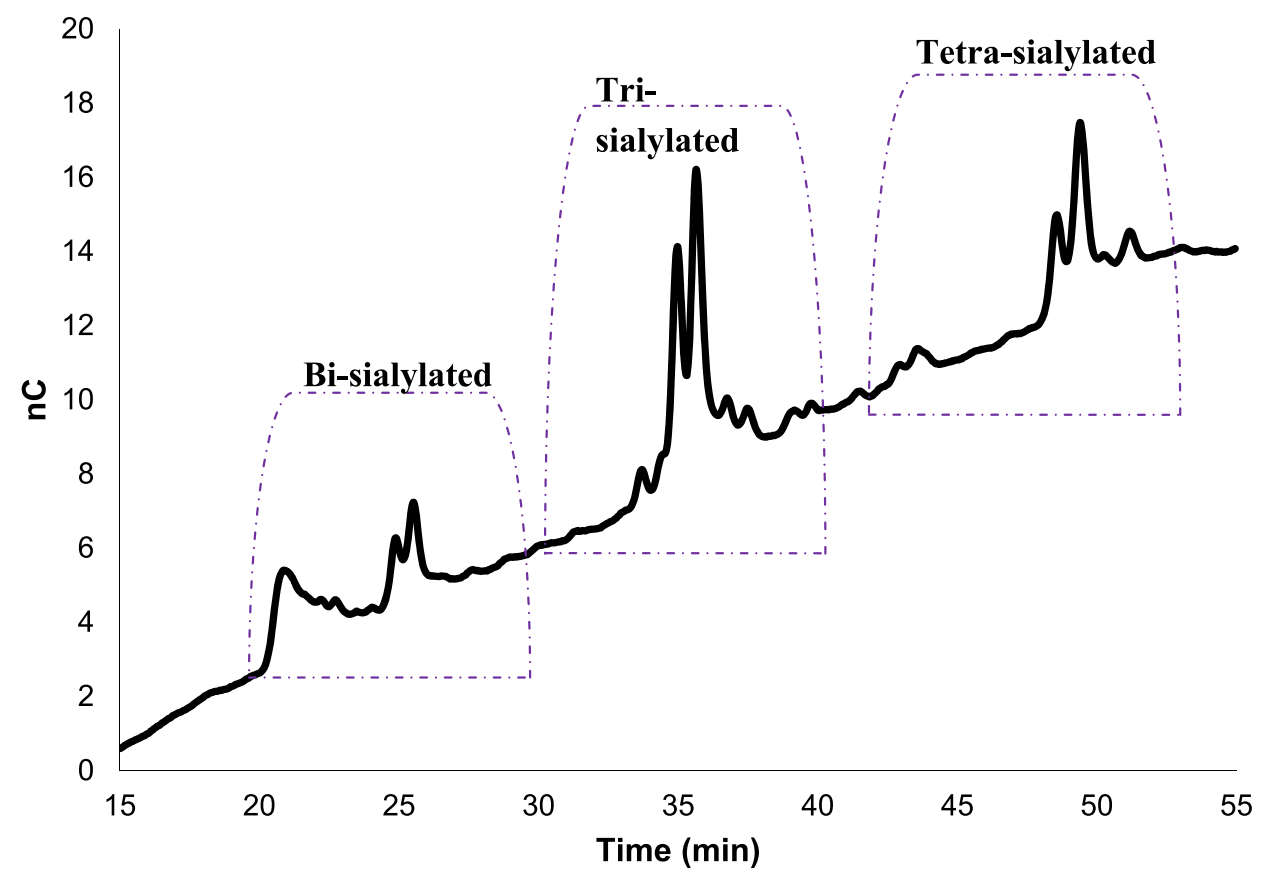

Figure 8. A HPAEC separation of a commercially available library of AGP oligosaccharides illustrating their separation into charged bands based on the number of sialic acid residues present at the chain terminus. 
The greater the number of sialic acids, the greater the overall negative charge resulting in a longer retention time. Typically bisialylated oligosaccharides elute between 20 and 30 minutes; trisialylated, 30 to 40 minutes and tetrasialylated 40-50 minutes (Figure 8). The oligosaccharide profiles for the AGP oligosaccharides from the clinical samples analysed in this study are shown in Figure 9.

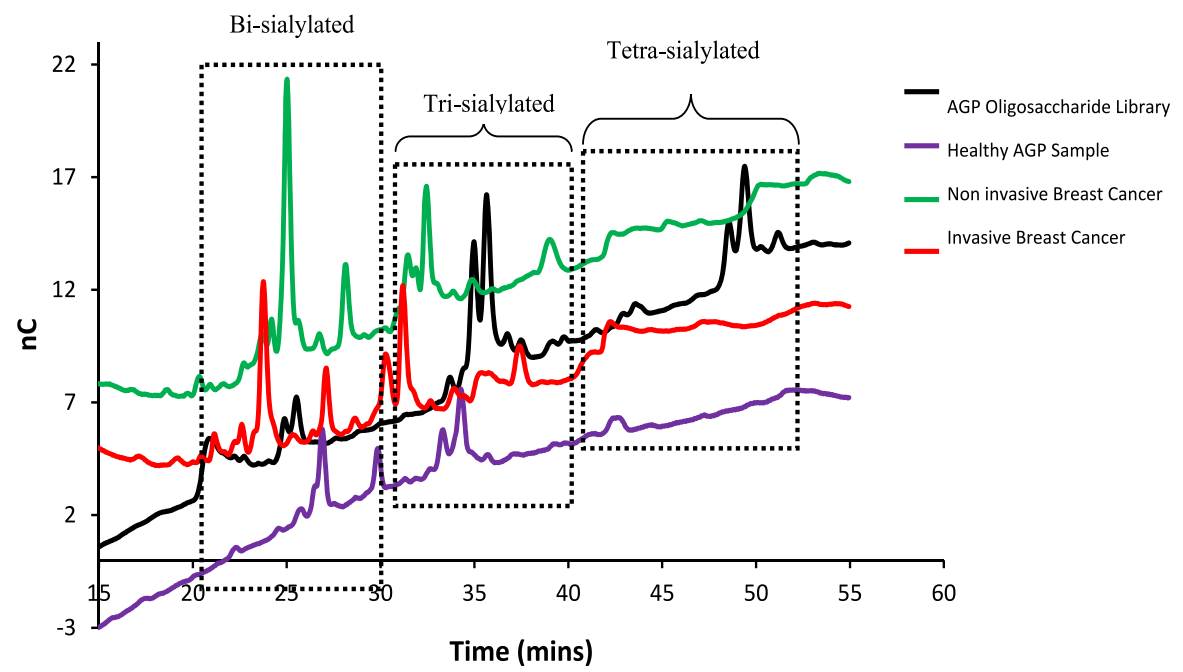

Figure 9. Representative oligosaccharide profiles of AGP, isolated from healthy volunteers (purple line), non malignant breast cancer (green line), malignant breast cancer (red line) were separated using high $\mathrm{pH}$ anion exchange chromatography and compared with a standard library of AGP oligosaccharides (black line).

The oligosaccharide profiles of AGP oligosaccharides from healthy volunteers, non malignant breast cancer and malignant breast cancer are comparable with the standard library (Figure 8) in that they both display peaks in the bi-, tri- and tetra-sialylated regions. Although qualitatively similar, the profiles for the breast cancer populations are noticeable for the earlier elution of the comparable peak regions. This reduction in retention is indicative of the presence of fucose on the oligosaccharide chains [49-50] which reinforces the results of the monosaccharide compositional analysis. Additionally, the two cancer populations appear to have fewer tetra-sialylated chains (elute 40-50 minutes) even if the presence of fucosylation is taken into account.

\section{Further research}

The oligosaccharide chains of each AGP in this study were released and analysed by high $\mathrm{pH}$ anion exchange chromatography [Figure 8] and showed differences that could be diagnostic and the basis of future research. Monosaccharide compositional analysis using HPAEC has highlighted the differences that exist between normal and breast disease 
populations. Specifically the presence of fucose, galactose and $\mathrm{N}$-acetylgalactosamine appears to be correlated with breast cancer malignancy. The presence of $\mathrm{N}$ acetylgalactosamine in the malignant population, together with its absence from the healthy and non-malignant populations suggests that the modification could form the basis of a serum biomarker for breast cancer malignancy with potential prognostic utility. HPAEC also provides useful information about the oligosaccharide structures present on AGP and allows the development of an oligosaccharide 'fingerprint' for each cohort of breast cancer patients.

\section{Conclusion}

Glycosylated molecules play major roles in oncogenesis, but their potential as cancer biomarkers remains unclear. Historically the reasons were twofold. Firstly, the large heterogeneity of structures arising from the cellular biosynthesis of glycans were not able to be detected and resolved by the analytical technology available at that point in time. Secondly the definitive diagnosis of cancerous disease normally required invasive techniques. These two hindrances have been addressed through the development of techniques such as high $\mathrm{pH}$ anion exchange chromatography (HPAEC) which now allows the sensitive (to picomole level) determination of glycosylated structures including linkage isomers of oligosaccharide chains. The solution to the second problem could lie in the development of tests based on the presence, in biological fluids, of glycosylated molecules which are disease-specific markers.

Alpha-1-acid glycoprotein is a prominent example of a molecule in which alterations in the structure of the surface oligosaccharides has provided some potential in the diagnosis and prognosis of physiological and pathophysiological conditions and determination of the effectiveness of treatments. There have been a vast number of studies concerned with quantifying the levels and characterising the microheterogeneity of AGP in a number of diseases [Reviewed in 7-9]. Variation in the branching of the chains, levels of fucose, sialic acid and the presence or absence of antigens such as sialyl Lewis $X$ are commonly reported and represent the potential exploitation of AGP heterogeneity as prognostic and diagnostic markers of disease [7].

In spite of major advances in detection and treatment, deaths from breast cancer are still high therefore there is a very definite requirement for the identification of a breast cancer specific biomarker to indicate the onset of the disease. Breast cancer is the most common cancer in women and currently diagnosis is reliant upon mammography and invasive techniques such as biopsy. There is no serum biomarker currently used in clinical practice for the detection and diagnosis of breast cancer. The discovery of a breast cancer-specific biomarker whose presence and/or altered expression in the serum precedes the appearance of a malignant mass would allow the development of a non-invasive serologic test and provide an invaluable screening tool in the assessment of high risk individuals. 
The current study has identified that the quantitative and qualitative changes in AGP glycosylation observed among patients with malignant and non malignant breast cancer may in fact, provide the basis of a serum biomarker with potential prognostic utility. The monosaccharide and oligosaccharide composition of the AGP glycosylation was found to differ between normal, non-invasive and invasive groups. From our results, fucose, Nacetylgalactosamine and galactose could be used as a screening test for early detection of breast cancer, and also as markers of breast carcinoma progression or "tumour load", as changes in the levels of the substances correlate with disease progression. Most importantly, $\mathrm{N}$-acetylgalactosamine could be used as a marker for the presence of malignancy in the breast, as $\mathrm{N}$-acetylgalactosamine is present only in the plasma of patients with malignant breast lesions. The implications of the use of $\mathrm{N}$-acetylgalactosamine as a marker for the presence of breast malignancy is profound; a definite diagnosis of breast carcinoma could be carried out by simple blood tests.

The current standard method of diagnosing early stages of breast cancer is mammography which, along with other imaging technology, requires expensive instrumentation. The use of blood tests to diagnose breast cancer would obviously have numerous advantages over mammography, and would prove to be an invaluable tool in the fight against breast cancer. The use of blood tests based on differences in AGP glycosylation for diagnosing breast cancer could be done inexpensively using ELISA. Mammography requires radiologists to review images, while screening for AGP markers can be performed by laboratory technicians with a high degree of automation. Mammography suffers from a relatively high degree of false diagnoses, while screening for $\mathrm{N}$-acetylgalactosamine appears to diagnose breast cancer with 100\% accuracy. Breast cancer has a high rate of survival if it is detected in its early stages. Unfortunately, large segments of the at-risk female population do not receive mammograms regularly, as a result of lack of access to mammography facilities, high cost, discomfort caused by the procedure, and potential or perceived risks of radiation exposure. The factors preventing women from receiving mammograms regularly would not be present with blood tests. Small blood samples could be taken from patients with minimal discomfort and cost in local medical centres. These samples could then be sent to large, centralised testing facilities for processing. The improved accessibility and convenience of this strategy over mammography would result in a greater segment of the at-risk female population receiving regular breast cancer screening, which would result in more early diagnoses, and therefore higher survival rates.

The use of blood tests that employ AGP alterations as markers of breast cancer has obvious commercial applications. These blood tests would likely become routine diagnostic procedures like the prostate-specific antigen (PSA) test. The potential market value of a breast cancer blood test would be comparable to that of the PSA test, which is about $\$ 1.5$ billion (USD) per year in the United States alone. Clearly, on an international scale, blood tests for diagnosing breast cancer would have a huge market value. In the event that AGP alterations are found to be markers of other cancers, the international market value of AGPbased blood tests would likely double or triple, reaching as high as $\$ 20$ billion (USD) per year. 


\section{Author details}

Kevin D. Smith* and Jennifer Behan

School of Life, Sport and Social Sciences,

Edinburgh Napier University, Sighthill Campus, Edinburgh, EH11 4BN, Scotland

Gerardine Matthews-Smith

School of Nursing, Midwifery and Social Care,

Edinburgh Napier University, Sighthill Campus, Edinburgh, EH11 4BN, Scotland

Anthony M. Magliocco

Department Chair Anatomic Pathology,

Esoteric Laboratory Services, H Lee Moffitt Cancer, Tampa, Florida, USA

\section{Acknowledgement}

Kevin Smith acknowledges funding from Friends for an Earlier Breast Cancer Test and Professor David George, University of Glasgow. Jennifer Behan was a funded by a Caledonian Scholarship from the Carnegie Trust for the Universities of Scotland.

\section{References}

[1] Kolarich D, Lepenies B, Seeberger PH. (2012) Glycomics, glycoproteomics and the immune system. Curr Opin Chem Biol. Accessed 2012 Jan 3. [Epub ahead of print].

[2] Hennet T. (2012) Diseases of glycosylation beyond classical congenital disorders of glycosylation. Biochim Biophys Acta. Accessed 2012 Feb 9. [Epub ahead of print]

[3] Dall'Olio F, Malagolini N, Trinchera M, Chiricolo M. (2012) Mechanisms of cancerassociated glycosylation changes. Front Biosci. 1;17:670-99.

[4] van Dijk W., Turner G A. and Mackiewicz A. (1994) Changes in glycosylation of acutephase proteins in health and disease: occurrence, regulation and function. Glycosylation and Disease. 1, 5-14.

[5] Adamczyk B, Tharmalingam T, Rudd PM. (2011) Glycans as cancer biomarkers. Biochim Biophys Acta. Accessed Dec 9. [Epub ahead of print].

[6] Narimatsu H, Sawaki H, Kuno A, Kaji H, Ito H, Ikehara Y. (2010) A strategy for discovery of cancer glyco-biomarkers in serum using newly developed technologies for glycoproteomics. FEBS J. 277(1):95-105.

[7] Ceciliani F, Pocacqua V. (2007) The acute phase protein $\alpha 1$-acid glycoprotein: a model for altered glycosylation during diseases. Current Protein and Peptide Science. 8, 91108.

[8] Fournier T, Medjoubi-N N , Porquet D. (2000) Alpha-1-acid glycoprotein. Biochimica et Biophysica Acta. 1482, 157-171.

[9] Israili Z H, Dayton G. (2001) Human alpha-1-glycoprotein and its interactions with drugs. Drug Met. Revs. 33, 161-235

\footnotetext{
* Corresponding Author
} 
[10] Duché J., Urien, S., Simon N, Malaurie E, Monnet I, Barré J. (2000) Expression of the genetic variants of human alpha-1-acid glycoprotein in cancer. Clin. Biochem. 33, 197202.

[11] Hervé F, Duché J, Jaurand M. (1998) Changes in expression and microheterogeneity of the genetic variants of human $\alpha 1$-acid glycoprotein in malignant mesothelioma. J. Chrom. B. 715, 111-23.

[12] Crestani B, Rolland C, Lardeux B, Fournier T, Bernuau D, Poüs C, Vissuzaine C, Li L, Aubier M. (1998) Inducible Expression of the $\alpha_{1}$-Acid Glycoprotein by Rat and Human Type II Alveolar Epithelial Cells. J. Immunol. 160, 4596-4605.

[13] Thompson DK, Haddow JE, Smith DE, Ritchie RF (1983). Elevated serum acute phase protein levels as predictors of disseminated breast cancer. Cancer; 51 2100-2104.

[14] Kailajarva M, Ahokoski O, Virtanen A, Salminen E, Irjala K. (2000). Early effects of adjuvant tamoxifen therapy on serum hormones, proteins and lipids. Anticancer Research, 20, 1323-1327.

[15] Dorssers LC, vander Flier S, Brinkman S, van Agthoven T, Veldscholte J, Berns EM, Klijn JG, Beex LV, Foekens JA (2001). Tamoxifen resistance in breast cancer: elucidating mechanisms. Drugs; 61, 1721-1733.

[16] Treuheit MJ, Costello CE, Halsall HB. (1992) Analysis of the five glycosylation sites of human $\alpha 1$-acid glycoprotein. Biochem. J.1992, 283, 105-112.

[17] Bayard B, Kerckaert JP (1980) Evidence for uniformity of the Carbohydrate Chains in Individual Glycoprotein Molecular Variants. Biochem Biophys Res Commun 95, 777784.

[18] Albani JR. (1997) Binding effect of progesterone on the dynamics of $\alpha 1$-acid glycoprotein. Biochimica et Biophysica Acta 1336, 349-359.

[19] Perkins SJ, Kerckaert J-P, Loucheux-lefebvre M. (1985) The shapes of biantennary and tri/tetraantennary $\alpha_{1}$-acid glycoprotein by small angle neutron and X-ray scattering. European J Biochem 147, 151-171.

[20] De Graaf TW, Van der Stelt ME, Anbergen MG, van Dijk W. (1993) Inflammationinduced expression of Sialyl Lewis X-containing glycan structures on $\alpha 1$-acid glycoprotein (orosomucoid) in human sera. J. Exp. Med. 177, 657-666.

[21] Fassbender K, Zimmerli W, Kissling R, Sobieska M, Aeschlimann A, Kellnar M, Müller W. (1991) Glycosylation of $\alpha 1$-acid glycoprotein in relation to duration of disease in acute and chronic infection and inflammation. Clinica Chimica Acta. 203, 315-328.

[22] Kratz E, Poland DCW, van Dijk W, Kątnik-Prastowska I. (2003) Alterations of branching and differential expression of sialic acid on alpha-1-acid glycoprotein in human seminal plasma. Clinica chimica acta: international journal of clinical chemistry. 331, 87-95.

[23] van den Heuvel MM, Poland DCW, De Graaf CS, Hoefsmit ECM, Postmus PE, Beelen RHJ, van Dijk W. (2000) The degree of branching of the glycans of $\alpha 1$-acid glycoprotein in asthma. American Journal of Respiratory and Critical Care Medicine. 161, 1972-1978.

[24] Smith KD, Pollacchi A, Field M, Watson J. (2002) The heterogeneity of the glycosylation of alpha-1-acid glycoprotein between the sera and synovial fluid in rheumatoid arthritis. Biomed. Chromatogr. 16, 261-266. 
[25] Elliott MA, Elliott HG, Gallagher K, McGuire J, Field M, Smith KD. (1997) An investigation into the Concanavalin A reactivity, fucosylation and oligosaccharide microheterogeneity of $\alpha_{1}$-acid glycoprotein expressed in the sera of rheumatoid arthritis patients. J. Chrom., Biomed. Appl. 688, 229-237.

[26] Jorgenson HG, Priest R, Smith K D. (1998) Modulation of sialyl Lewis X dependent cell binding to E-selectin by glycoforms of alpha-1-acid glycoprotein expressed in rheumatoid arthritis. Biomed. Chromatogr. $12: 1-7$.

[27] Dage JL, Ackermann B, and Halsall HB. (1998) Site localization of sialyl Lewis ${ }^{\mathrm{X}}$ antigen on $\alpha_{1}$-acid glycoprotein by high performance liquid chromatography-electrospray mass spectrometry. Glycobiology. 8, 755-760.

[28] van Dijk W, Koeleman C, Van het Hof B, Poland D, Jakobs C, Jaeken, J. (1998) Increased $\alpha 3$-fucosylation of $\alpha_{1}$-acid glycoprotein in patients with congenital disorder of glycosylation type IA (CDG-Ia) Trends Glycosci Glycotecnol.10, 235-245.

[29] Havenaar EC, Hoff RC, van den Eijnden DH, van Dijk W. Severe rheumatoid arthritis prohibits the pregnancy-induced decrease in $\alpha 3$-fucosylation of $\alpha_{1}-$ acid glycoprotein. Glycoconjugate J 1998, 15, 389-395.

[30] Poland DC, Schalkwijk CG, Stehouwer CD, Koeleman C.A, van het Hof B, van Dijk W. (2001) Increased alpha3-fucosylation of alpha1-acid glycoprotein in Type I diabetic patients is related to vascular function. Glycoconjugate Journal. 2001, 18, 261-268.

[31] Rydén I, Påhlsson P, Lundblad A., Skogh T. (2002) Fucosylation of $\alpha 1$-acid glycoprotein (orosomucoid) compared with traditional biochemical markers of inflammation in recent onset rheumatoid arthritis. Clinica Chimica Acta. 317, 221-229.

[32] Elliott MA, Jørgensen HG, Smith KD. (1998) Hypersialylation of $\alpha_{1}$-acid glycoprotein in rheumatoid arthritis. Pharmacy and Pharmacology Communications. 4, 545-54.

[33] Pawiłowicz, M., Hirnle, L. and Kątnik-Prastowska, I. (2006) Alterations of N-glycan branching and expression of sialic acid on amniotic fluid alpha-1-acid glycoprotein derived from second and third trimesters of normal and prolonged pregnancies. Clinica Chimica Acta. 367, 86-92.

[34] Anderson N, Pollacchi A, Hayes P, Therapondos G., Newsome P, Boyter A, and Smith K. (2002) A preliminary evaluation of the differences in the glycosylation of alpha-1acid glycoprotein between individual liver diseases. Biomed. Chromatogr. 16, 365-372.

[35] Rydén I, Påhlsson P, Lindgren S. (2002) Diagnostic accuracy of alpha (1)-acid glycoprotein fucosylation for liver cirrhosis in patients undergoing hepatic biopsy. Clinical chemistry. 48, 2195-2201.

[36] Mooney P, Hayes P, Smith K. (2006) The putative use of $\alpha$-1-acid glycoprotein as a noninvasive marker of fibrosis. Biomed. Chromatogr. 20, 1351-1358.

[37] Adam P, Sobek O, Táborsk L, Hildebrand T, Tutterová, Žáček P. (2003) CSF and serum orosomucoid ( $\alpha$-1-acid glycoprotein) in patients with multiple sclerosis: a comparison among particular subgroups of MS patients. Clinica Chimica Acta. 334, 107-110.

[38] French D, Watson J, McCahill B, Taggart I, Smith KD. (2002) A preliminary evaluation of the functional significance of alpha-1-acid glycoprotein glycosylation on wound healing. Biomedical Chromatography. 16, 412-419. 
[39] Pos O, van Dijk W, Ladiges N, Linthorst C, Sala M, van Tiel D, Boers W. (1998) Glycosylation of four acute-phase glycoproteins secreted by rat liver cells in vivo and in vitro. Effects of inflammation and dexamethasone Eur J Cell Biol 46, 121-128.

[40] Brinkman-Van der Linden ECM, Havenaar EC, Van Ommen ECR, Van Kamp GJ, Gooren LJG, van Dijk W. (1996) Oral estrogen treatment induces a decrease in expression of sialyl Lewis $\mathrm{x}$ on $\alpha 1$-acid glycoprotein in females and male-to-female transsexuals. Glycobiology. 6, 407-412.

[41] Hansen JS, Larsen VA, Bøg-Hansen TC. (1984) The microheterogeneity of $\alpha 1$-acid glycoprotein in inflammatory lung disease, cancer of the lung and normal health. Clinica Chimica Acta. 138, 41-47.

[42] Bleasby AJ, Knowles JC, Cooke NJ. (1985) Microheterogeneity of $\alpha 1$-acid glycoprotein: lack of discrimination between benign and malignant inflammatory disease of the lung. Clinica Chimica Acta. 150, 231-235.

[43] Fujii M, Takahashi N, Hayashi H, Furusho T, Matsunaga K, Yoshikumi C. (1988) Comparative study of $\alpha_{1}$-acid glycoprotein molecular variants in ascitic fluid of cancer and non-cancer patients. Anticancer Research. 8, 303-306

[44] Moule SK, Peak M, Thompson S, Turner GA. (1987) Studies of the sialylation and microheterogeneity of human serum $\alpha 1$-acid glycoprotein in health and disease. Clinica Chimica Acta. 166, 177-185.

[45] Turner GA, Skillen AW, Buamah P, Guthrie D, Welsh J, Harrison J, Kowalski A. (1985) Relation between raised concentrations of fucose, sialic acid and the acute phase proteins in serum from patients with cancer: choosing suitable serum glycoprotein markers. Journal of Clinical Pathology. 38, 588-592.

[46] Hashimoto S, Asao T, Takahashi J, Yagihashi Y, Nishimura T, Saniabadi A R, Poland DCW, van Dijk W, Kuwano H, Kochibe N Yazawa S. (2004) $\alpha 1$-acid glycoprotein fucosylation as a marker of carcinoma progression and prognosis. Cancer. 101, 28252836.

[47] van Dijk W, Havenaar EC, Brinkman-van der Linden ECM. (1995) $\alpha 1$-acid glycoprotein (orosomucoid): pathophysiological changes in glycosylation in relation to its function. Glycoconjugate J. 12, 227-233.

[48] Sakorafas GH, Farley DR (2003) Optimal management of ductal carcinoma in situ of the breast. Surg Oncol 2003: 12:221-240.

[49] Behan J, Smith, K.D. (2011) The analysis of glycosylation: A continued need for high pH anion exchange chromatography. Biomed. Chrom. 25, 39-46.

[50] Smith KD. (1997) Structural Elucidation of the N-linked Oligosaccharides of Glycoproteins using High $\mathrm{pH}$ Anion Exchange Chromatography. Advances in Macromolecular Carbohydrate Research 1, 65-91. 